\title{
Impacto psicológico e fatores associados à pandemia da COVID-19 e ao distanciamento social em Minas Gerais, Brasil: Estudo transversal
}

Psychological impact and associated factors with the COVID-19 pandemic and social distancing in Minas Gerais, Brazil: Cross-sectional study

Impacto psicológico y factores asociados con la pandemia del COVID-19 y el distanciamiento social en Minas Gerais, Brasil: Estudio transversal

Recebido: 24/06/2021 | Revisado: 01/07/2021 | Aceito: 06/07/2021 | Publicado: 16/07/2021

Tâmaro Chagas Mendes

ORCID: https://orcid.org/0000-0003-0645-6706 Universidade Federal dos Vales do Jequitinhonha e Mucuri, Brasil E-mail: tamaro.mendes@ufvjm.edu.br Camila de Lima

ORCID: https://orcid.org/0000-0001-9099-4376 Universidade Federal dos Vales do Jequitinhonha e Mucuri, Brasil E-mail: camila.delima@ufvjm.edu.br

Bruno Henrique Melo Fernandes

ORCID: https://orcid.org/0000-0002-6015-9756 Universidade Federal dos Vales do Jequitinhonha e Mucuri, Brasil E-mail: bruno.melo@ufvjm.edu.br

Celina de Vasconcelos Leite

ORCID: https://orcid.org/0000-0002-9670-4963 Universidade Federal dos Vales do Jequitinhonha e Mucuri, Brasil E-mail: celina.vasconcelos@ufvjm.edu.br

Daniel Santos Gonçalves

ORCID: https://orcid.org/0000-0002-0294-8503

Universidade Federal dos Vales do Jequitinhonha e Mucuri, Brasil E-mail: goncalves.daniel@ufvjm.edu.br

Júlia de Souza Brasil da Silva

ORCID: https://orcid.org/0000-0001-6472-7207

Universidade Federal dos Vales do Jequitinhonha e Mucuri, Brasil E-mail: julia.brasil@ufvjm.edu.br

Ellen Cristina Rodrigues Neves

ORCID: https://orcid.org/0000-0002-0771-3914 Universidade Federal dos Vales do Jequitinhonha e Mucuri, Brasil E-mail: ellen.neves@ufvjm.edu.br

Larissa Rangel Souto

ORCID: https://orcid.org/0000-0002-0571-6978

Universidade Federal dos Vales do Jequitinhonha e Mucuri, Brasil E-mail: larissa.rangel@ufvjm.edu.br

Laura Luísa de Oliveira Elias

ORCID: https://orcid.org/0000-0002-4361-7356

Universidade Federal dos Vales do Jequitinhonha e Mucuri, Brasil

E-mail: laura.oliveira@ufvjm.edu.br

Margarida Maria Tavares Lacerda de Medeiros

ORCID: https://orcid.org/0000-0002-9162-2053

Universidade Federal dos Vales do Jequitinhonha e Mucuri, Brasil

E-mail: margarida.medeiros@ufvjm.edu.br

Raíssa Lisboa Ramos

ORCID: https://orcid.org/0000-0002-9109-4326

Universidade Federal dos Vales do Jequitinhonha e Mucuri, Brasil

E-mail: raissa.ramos@ufvjm.edu.br

\begin{abstract}
Resumo
O surgimento de uma pandemia pode gerar impactos importantes à saúde mental das populações afetadas. O objetivo desta pesquisa foi identificar o impacto psicológico e os fatores associados durante um período da pandemia da COVID19 e do distanciamento social em uma amostra dos habitantes do estado de Minas Gerais, Brasil. A pesquisa possuiu caráter transversal e descritivo com abordagem quantitativa. Utilizou-se como instrumentos de pesquisa um questionário sociodemográfico e o Self Report Questionnaire (SRQ-20) para avaliar a suspeita de Transtornos Mentais Comuns (TMC). A pesquisa obteve 619 respostas por meio de um questionário online. A prevalência de TMC na amostra foi de 42,6\% (IC95\%: 38,7-46,5). Encontrou-se associação entre TMC e indivíduos jovens, mulheres, solteiros, estudantes
\end{abstract}


universitários, participantes que possuíam um transtorno mental diagnosticado, que tiveram a rotina, os estudos e a renda familiar afetada e estavam muito insatisfeitos com as informações sobre a COVID-19 divulgadas pelas autoridades públicas de saúde e muito preocupados com a influência que a COVID-19 terá sobre seus estudos. A pandemia da COVID-19 promoveu um relevante impacto psicológico nesta amostra da população mineira.

Palavras-chave: COVID-19; Pandemias; Saúde mental.

\begin{abstract}
The emergence of a pandemic can have important impacts on the mental health of the affected populations. The objective of this research was to identify the psychological impact and associated factors during a period of the COVID19 pandemic and social distance in a sample of the inhabitants of the state of Minas Gerais, Brazil. This cross-sectional study had a descriptive character with a quantitative approach. We used a sociodemographic questionnaire and the Self Report Questionnaire (SRQ-20) to assess the suspicion of Common Mental Disorders (CMD). The survey obtained 619 responses through an online questionnaire. The prevalence of CMD in the sample was 42.6\% (CI95\%: 38.7-46.5). We found associations between CMD and young participants, women, singles, college students, those participants who had a diagnosed mental disorder, who had their routine, studies and family income affected and were very dissatisfied with the information about COVID-19 released by the public health authorities and very concerned about the influence that COVID-19 will have on their studies. The COVID-19 pandemic promoted a relevant psychological impact in this sample of the population of Minas Gerais.
\end{abstract}

Keywords: COVID-19; Pandemics; Mental health.

\title{
Resumen
}

La aparición de una pandemia puede tener impactos importantes en la salud mental de las poblaciones afectadas. El objetivo de esta investigación fue identificar el impacto psicológico y los factores asociados durante un período de la pandemia del COVID-19 y del distanciamiento social en una muestra de habitantes del estado de Minas Gerais, Brasil. La investigación tuvo un carácter transversal y descriptivo con un enfoque cuantitativo. Se utilizó un cuestionario sociodemográfico y el Self Report Questionnaire (SRQ-20) como instrumentos de investigación para evaluar la sospecha de Trastornos Mentales Comunes (TMC). La encuesta obtuvo 619 respuestas a través de un cuestionario online. La prevalencia de TMC en la muestra fue del 42,6\% (IC95\%: 38,7-46,5). Se encontró asociación entre TMC y personas jóvenes, mujeres, solteros, estudiantes universitarios, participantes que tenían un trastorno mental diagnosticado, que tenían su rutina, educación e ingresos familiares afectados y estaban muy insatisfechos con la información sobre el COVID-19 divulgada por las autoridades sanitarias y muy preocupados por la influencia que tendrá el COVID-19 en sus estudios. La pandemia del COVID-19 tuvo un impacto psicológico significativo en esta muestra de la población de Minas Gerais

Palabras clave: COVID-19; Pandemias; Salud mental.

\section{Introdução}

O surgimento de uma pandemia pode gerar impactos importantes à saúde mental das populações afetadas. O surto da Síndrome Respiratória Aguda Grave (SARS) que em 2002-2003 afetou a China, Hong Kong e outros países, esteve entre os primeiros surtos que tiveram aspectos relacionados à saúde mental estudados (Huremovic, 2019). Demonstrou-se que indivíduos que foram colocados em quarentena ou indiretamente expostos à SARS durante a epidemia tendiam a apresentar sintomas de depressão possivelmente devido ao impacto da doença, à desaceleração econômica, às más condições de saúde e ao suporte social inadequado (Ko, Yen, Yen, \& Yang, 2006).

Em dezembro de 2019, na cidade de Wuhan, província de Hubei, na China, foi identificada uma pneumonia de etiologia desconhecida cujo agente causador foi nomeado Síndrome Respiratória Aguda Grave Coronavírus 2 (SARS-CoV-2) (Sohrabi et al., 2020). Posteriormente, a Organização Mundial de Saúde (OMS) nomeou a doença de COVID-19 e declarou em 31 de janeiro de 2020 uma emergência de saúde pública de interesse internacional (World Health Organization, 2020).

Assim como ocorreu com a SARS, algumas pesquisas identificaram significativo impacto psicológico neste momento da pandemia da COVID-19. Na China, primeiro país afetado pela doença, demonstrou-se que mulheres, estudantes, pessoas que apresentaram sintomas da COVID-19, situação de saúde frágil e histórico de doenças crônicas, experimentaram maior impacto psicológico e níveis mais altos de estresse, ansiedade e depressão (Wang et al., 2020). Outras pesquisas incluindo países do ocidente como Estados Unidos, Espanha e Itália, encontraram taxas relativamente altas de sintomas de ansiedade, depressão, transtorno de estresse pós-traumático, sofrimento psicológico e estresse cujos fatores de risco associados incluíam sexo feminino, 
faixa etária mais jovem, presença de doenças crônicas/psiquiátricas, desemprego, ser estudante e exposição frequente às mídias sociais e notícias sobre a COVID-19 (Xiong et al., 2020).

Sobre o impacto psicológico na população brasileira, há indicativos de significativa prevalência de ansiedade, depressão e estresse associados ao sexo feminino, jovens, baixa escolaridade, baixa renda e longo período em distanciamento social (Goularte et al., 2021). Entretanto, as características sociais, econômicas e culturais dos estados brasileiros podem influenciar que o impacto psicológico da pandemia seja experenciado de modo diferente na população. A pesquisa de Duarte et al. (2020) com uma amostra do Rio Grande do Sul, por exemplo, optou por uma investigação de algumas variáveis que tiveram relevância para o recorte local, como profissão, existência de transtorno mental prévio e ser do grupo de risco para a COVID-19. Deste modo, pode ser relevante investigar sobre o impacto da pandemia na saúde mental da população em cada estado. Esta pesquisa se propõe a colaborar pesquisando uma amostra da população do estado de Minas Gerais.

Assim como ocorreu no cenário nacional, o estado de Minas Gerais também foi fortemente afetado pela COVID-19 e reconheceu o estado de calamidade pública devido à doença (Minas Gerais, 2020). Considerando o quadro epidemiológico e as medidas restritivas de circulação e aglomeração, torna-se relevante avaliar as condições que afetam a saúde mental da população mineira. Assim, esta pesquisa possuiu o objetivo de identificar o impacto psicológico e os fatores associados durante um período da pandemia da COVID-19 e do distanciamento social em uma amostra dos habitantes do estado de Minas Gerais.

\section{Metodologia}

\section{Caracterização do estudo}

A pesquisa possuiu caráter transversal e descritivo com abordagem quantitativa. Este estudo utilizou dados do projeto "Impacto psicológico e fatores associados durante a pandemia da COVID-19 e do distanciamento social entre estudantes universitários, trabalhadores da área da saúde e população geral brasileira". Utilizou-se os dados dos 619 participantes maiores de 18 anos e residentes no estado de Minas Gerais, localidade com maior número de respondentes na pesquisa.

\section{Coleta de dados}

Elaborou-se para a coleta de dados um questionário online no site Formulários Google que foi distribuído via e-mail e redes sociais. Utilizou-se a técnica snowball, mesma utilizada por Wang et al. (2020), solicitando que os participantes compartilhassem o questionário com outras pessoas. O questionário ficou disponível para receber respostas entre os dias 16/07/2020 e 16/08/2020.

\section{Instrumentos}

Foram utilizados como instrumentos de pesquisa um questionário elaborado pelos autores contendo variáveis sociodemográficas e elementos acerca da COVID-19 e do distanciamento social, identificados a partir de revisão de literatura sobre o impacto psicológico da COVID-19 na população geral e o Self Report Questionnaire (SRQ-20).

O SRQ-20 foi desenvolvido pela OMS, validado no Brasil por Mari e Williams (1985) e avalia a presença de Transtornos Mentais Comuns (TMC), que são estados mistos de depressão e ansiedade e de outros sintomas como insônia, fadiga, irritabilidade, esquecimento, dificuldade de concentração e queixas somáticas (Graner \& Cerqueira, 2019). O questionário possui 20 questões com respostas do tipo "sim" e "não". Atribuiu-se um ponto para cada "sim", permanecendo a pontuação total entre 0 e 20. Os sintomas avaliados referem-se aos últimos 30 dias e considera-se como suspeito de TMC participantes que obtiveram pontuação igual ou maior do que 7, ponto de corte já utilizado na população brasileira (K. O. B. Santos, Araújo, Pinho, \& Silva, 2010). Pesquisas anteriores demonstraram que o SRQ-20 mostrou-se adequado para avaliar o impacto psicológico no contexto da pandemia da COVID-19 (Duarte et al., 2020; Pimentel, Figueiredo, Mattos, \& Barreto, 2020). 


\section{Aspectos éticos}

A pesquisa recebeu aprovação pela Comissão Nacional de Ética em Pesquisa (Conep) e pelo Comitê de Ética em Pesquisa (CEP) da Universidade Federal dos Vales do Jequitinhonha e Mucuri (UFVJM), parecer nº 4.378.996.

\section{Análise dos dados}

A análise estatística utilizou medidas de tendência central e dispersão (média e desvio padrão) para as variáveis contínuas e distribuições de frequência absoluta e relativa para as variáveis categóricas. Na análise bivariada, utilizou-se o teste qui-quadrado de Pearson ou o Teste Exato de Fisher a fim de verificar as associações entre a variável dependente suspeita de TMC e as variáveis independentes. As razões de prevalência e seus intervalos de 95\% de confiança (IC95\%) foram estimados pela regressão de Poisson com variâncias robustas, método adequado para estudos transversais (Coutinho, Scazufca, \& Menezes, 2008). Posteriormente, foram construídos modelos multivariados com a regressão de Poisson com variâncias robustas adotandose a entrada hierárquica. No modelo 1 foram incluídas as variáveis sociodemográficas que apresentaram nível de significância com $\mathrm{p}<0,20$ na análise bivariada, tendo permanecido no modelo aquelas com valor de $\mathrm{p}<0,05$. No modelo 2 , foram incluídas as variáveis sobre a COVID-19 e o distanciamento social, utilizando os mesmos valores de significância do modelo anterior. Este modelo de análise baseou-se em um estudo epidemiológico realizado por Chagas et al. (2019). Para todos os testes, adotou-se como nível de significância estatística o valor de $\mathrm{p} \leq 0,05$. Todas as análises estatísticas foram executadas no programa Jamovi versão 1.6.15 (The Jamovi project, 2021).

\section{Resultados}

Participaram da pesquisa 619 pessoas com idade de 18 a 72 anos (média $=34,8$; desvio padrão= 12,6). A maioria dos participantes estava na faixa etária de 18 a 24 anos (172; 27,8\%); era do sexo feminino (443; 71,6\%); solteiros (341; 55,1\%); brancos $(282 ; 45,6 \%)$; com ensino superior incompleto (179; 28,9\%); residindo com 03 pessoas $(173 ; 27,9 \%)$ e não morava com crianças $(442 ; 71,4 \%)$ ou idosos $(491 ; 79,3 \%)$. Em relação à ocupação principal, participaram profissionais de outras áreas (educação, administração, judiciário, tecnologia, aposentados, autônomos, setor de serviços, serviço público, artes etc.) (229; $37 \%$ ), estudantes universitários (209; 33,8\%), profissionais da área da saúde que estão na linha de frente de combate à COVID19 (médicos, enfermeiros, técnicos de enfermagem, psicólogos, profissionais das áreas administrativas do setor de saúde etc.) (90; 14,5\%), profissionais de serviços essenciais (limpeza pública, transporte, segurança, alimentação, farmácia, banco etc.) (80; $12,9 \%)$ e em menor número, pessoas que não declaram nenhuma ocupação (11; 1,8\%). A maioria dos participantes respondeu que a renda familiar estava na faixa de 1 a 5 salários mínimos (SM) (346; 55,9\%) e que essa renda diminuiu durante o período da pandemia pesquisado $(313 ; 50,6 \%)$. Em relação à saúde mental, houve predomínio de participantes que não possuíam diagnóstico de transtorno mental $(477 ; 77,1 \%)$.

Sobre a COVID-19 e o distanciamento social, a maioria dos participantes não fez teste para diagnosticar a doença (530; $85,6 \%)$, mas aqueles fizeram $(89 ; 14,4 \%)$, o resultado negativo prevaleceu $(67 ; 75,3 \%)$. Em relação à saída de casa durante o distanciamento social, os participantes majoritariamente afirmaram que saem de casa de 1 a 2 vezes na semana $(219 ; 35,4 \%)$. Predominou entre os participantes a opinião de que a rotina foi muito afetada devido ao distanciamento social $(301 ; 48,6 \%)$. Quanto à modalidade de trabalho, compôs a amostra participantes que não estavam trabalhando atualmente (220; 35,5\%), estavam indo para o trabalho presencial diariamente $(223 ; 36 \%)$ ou estavam em casa no teletrabalho $(176 ; 28,4 \%)$. Sobre a satisfação em relação às informações divulgadas pelas autoridades públicas de saúde, prevaleceu os participantes insatisfeitos (191; 30,9\%). Sobre suas preocupações, os participantes estavam muito preocupados com a influência da COVID-19 na economia do Brasil $(359 ; 58 \%)$ e nos estudos $(244 ; 39,4 \%)$.

Em relação ao impacto psicológico, 264 participantes $(42,6 \%$; IC95\%: 38,7-46,5) foram classificados com suspeita de 
TMC. A média de pontuação no SRQ-20 foi de 6,23 (desvio padrão= 5,06). Em relação aos sintomas apresentados no questionário, sentir-se nervoso, tenso ou preocupado foi o mais citado pelos participantes (69,9\%). O Gráfico 1 demonstra as frequências dos sintomas encontrados na amostra.

Gráfico 1: Frequência relativa de sintomas de TMC na amostra.

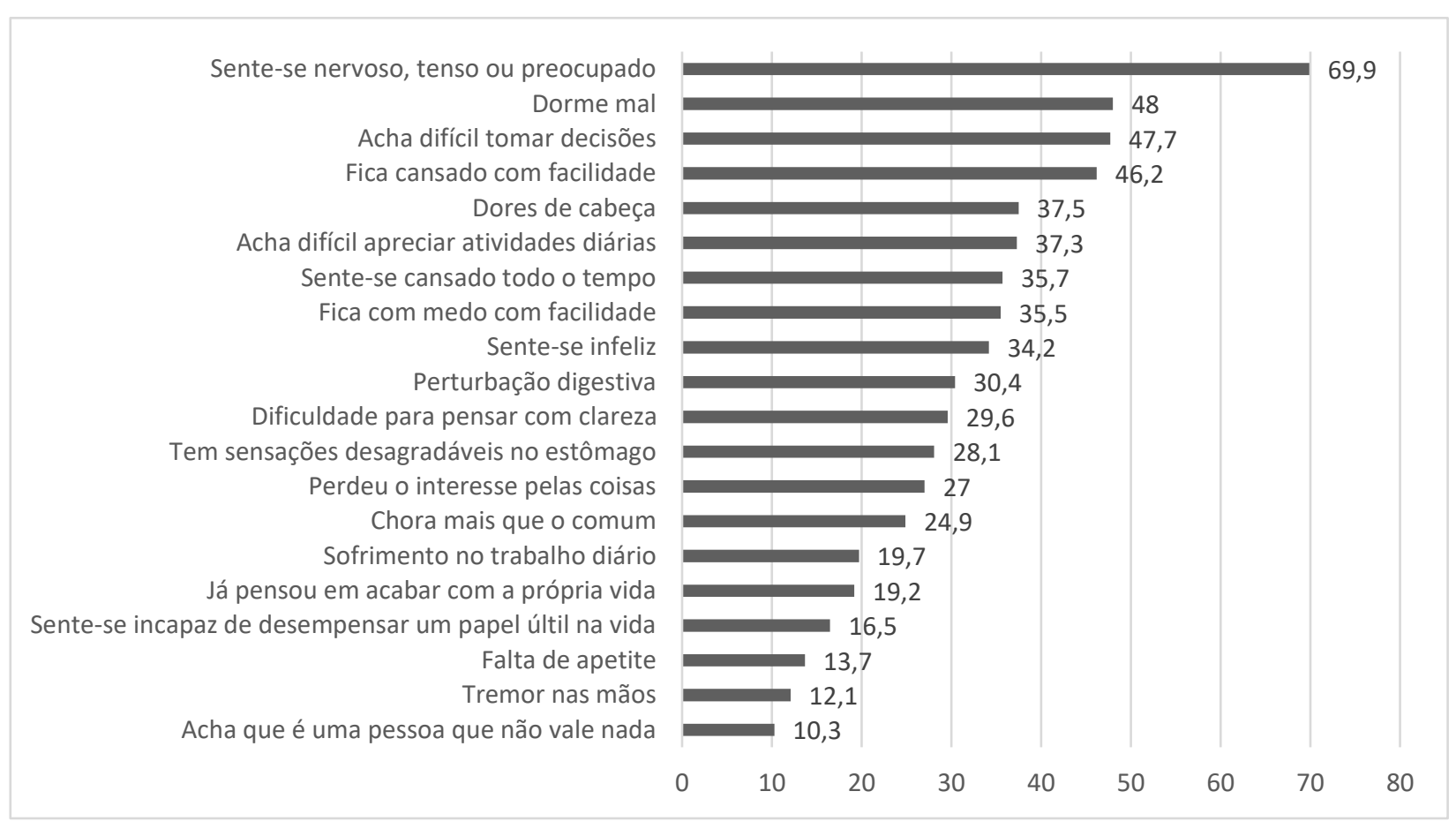

Fonte: Autoria própria (2021).

Foi significativa a associação entre TMC e as variáveis sociodemográficas faixa etária, sexo, estado civil, escolaridade, moradia, ocupação, renda familiar, alteração na renda familiar e existência de transtorno mental. Nestas variáveis, a prevalência de TMC foi maior nos jovens de 18 a 24 anos (107; 62,2\%); mulheres (217; 49\%); solteiros (181; 53,1\%); com ensino superior incompleto (104; 58,1\%); estudantes universitários (126; 60,3\%); aqueles que moravam com 03 pessoas $(68 ; 73,6 \%)$, com renda familiar menor do que 5 SM $(164 ; 47,4 \%)$; cuja renda familiar diminuiu o período da pandemia $(149 ; 47,6 \%)$ e nas pessoas que possuíam um transtorno mental diagnosticado $(111 ; 78,2 \%)$. A Tabela 1 exibe a associação entre as variáveis sociodemográficas e a suspeita de TMC. 
Research, Society and Development, v. 10, n. 8, e48310817541, 2021

(CC BY 4.0) | ISSN 2525-3409 | DOI: http://dx.doi.org/10.33448/rsd-v10i8.17541

Tabela 1: Associação entre variáveis sociodemográficas e suspeita de TMC.

\begin{tabular}{|c|c|c|c|c|}
\hline Variável & $\begin{array}{c}\mathbf{N} \\
\mathrm{n}(\%)\end{array}$ & $\begin{array}{l}\text { TMC } \\
\text { n (\%) }\end{array}$ & RP (IC95\%) & p-valor* \\
\hline \multicolumn{5}{|l|}{ Faixa etária } \\
\hline $18-24$ & $172(27,8)$ & $107(62,2)$ & 1 & \multirow{5}{*}{$<0,001$} \\
\hline $25-32$ & $142(22,9)$ & $72(50,7)$ & $0,81(0,64-1,02)$ & \\
\hline $33-45$ & $157(25,4)$ & $52(33,1)$ & $0,53(0,41-0,68)$ & \\
\hline $46-59$ & $118(19,1)$ & $26(22)$ & $0,35(0,25-0,48)$ & \\
\hline $60-72$ & $23(3,7)$ & $5(21,7)$ & $0,34(0,16-0,64)$ & \\
\hline \multicolumn{5}{|l|}{ Sexo } \\
\hline Feminino & $443(71,6)$ & $217(49)$ & $1,83(1,45-2,34)$ & \multirow{2}{*}{$<0,001$} \\
\hline Masculino & $176(28,4)$ & $47(26,7)$ & 1 & \\
\hline \multicolumn{5}{|l|}{ Estado civil } \\
\hline Solteiro & $341(55,1)$ & $181(53,1)$ & 1 & \multirow{4}{*}{$<0,001$} \\
\hline Casados & $232(37,5)$ & $70(30,2)$ & $0,56(0,45-0,69)$ & \\
\hline Divorciado & $34(5,5)$ & $9(26,5)$ & $0,49(0,28-0,80)$ & \\
\hline Viúvo & $12(1,9)$ & $4(33,3)$ & $0,62(0,26-1,23)$ & \\
\hline \multicolumn{5}{|l|}{ Cor } \\
\hline Branco & $282(45,6)$ & $118(41,8)$ & 1 & \multirow{4}{*}{0,467} \\
\hline Pardo & $267(43,1)$ & $110(41,2)$ & $0,98(0,80-1,19)$ & \\
\hline Negro & $44(7,1)$ & $23(52,3)$ & $1,24(0,87-1,73)$ & \\
\hline Amarelo & $26(4,2)$ & $13(50)$ & $1,19(0,75-1,80)$ & \\
\hline \multicolumn{5}{|l|}{ Escolaridade } \\
\hline Fundamental & $16(2,6)$ & $7(43,8)$ & $1,54(0,80-2,72)$ & \multirow{5}{*}{$<0,001$} \\
\hline Médio & $94(15,2)$ & $43(35,7)$ & $1,62(1,18-2,21)$ & \\
\hline Superior incompleto & $179(28,9)$ & $104(58,1)$ & $2,05(1,59-2,68)$ & \\
\hline Superior completo & $160(25,8)$ & $62(38,8)$ & $1,37(1,03-1,83)$ & \\
\hline Pós-graduado & $170(27,5)$ & $48(28,2)$ & 1 & \\
\hline \multicolumn{5}{|l|}{ Moradia } \\
\hline Moro sozinho & $56(9)$ & $20(23,8)$ & 1 & \multirow{5}{*}{0,006} \\
\hline 02 pessoas & $152(24,6)$ & $58(64)$ & $1,06(0,73-1,59)$ & \\
\hline 03 pessoas & $173(27,9)$ & $68(73,6)$ & $1,10(0,76-1,63)$ & \\
\hline 04 pessoas & $161(26)$ & $70(68,5)$ & $1,21(0,84-1,80)$ & \\
\hline 5 ou mais pessoas & $76(12,3)$ & $47(32,3)$ & $1,73(1,17-2,61)$ & \\
\hline \multicolumn{5}{|l|}{ Criança em casa } \\
\hline Não & $441(71,4)$ & $195(44,1)$ & 1 & \multirow{2}{*}{0,243} \\
\hline Sim & $177(28,6)$ & $69(39)$ & $0,88(0,71-1,08)$ & \\
\hline \multicolumn{5}{|l|}{ Idoso em casa } \\
\hline Não & $491(79,3)$ & $211(43)$ & 1 & \multirow{2}{*}{0,749} \\
\hline Sim & $128(20,7)$ & $53(41,4)$ & $0,96(0,76-1,20)$ & \\
\hline \multicolumn{5}{|l|}{ Ocupação principal } \\
\hline Profissional de outras áreas & $229(37)$ & $78(34,1)$ & 1 & \multirow{5}{*}{$<0,001$} \\
\hline Estudante universitário & $209(33,8)$ & $126(60,3)$ & $1,77(1,43-2,19)$ & \\
\hline Profissional de saúde & $90(14,5)$ & $27(30)$ & $0,88(0,62-1,21)$ & \\
\hline Profissional de serviços essenciais & $80(12,9)$ & $26(32,5)$ & $0,95(0,67-1,32)$ & \\
\hline Não está trabalhando atualmente & $11(1,8)$ & $7(63,6)$ & $1,86(0,98-3,22)$ & \\
\hline Renda familiar & & & & \\
\hline$<1 \mathrm{SM}$ & $22(3,6)$ & $12(54,5)$ & 1 & \\
\hline$>1 \mathrm{a}<5 \mathrm{SM}$ & $346(55,9)$ & $164(47,4)$ & $0,86(0,57-1,40)$ & \\
\hline$>5 \mathrm{a}<10 \mathrm{SM}$ & $133(21,5)$ & $47(35,3)$ & $0,64(0,40-1,07)$ & $<0,001$ \\
\hline$>10 \mathrm{SM}$ & $83(13,4)$ & $21(25,3)$ & $0,46(0,27-0,80)$ & \\
\hline Não desejou informar & $35(5,7)$ & $20(57,1)$ & $1,04(0,61-1,83)$ & \\
\hline Alteração na renda familiar & & & & \\
\hline Não foi afetada & $292(47,2)$ & $108(37)$ & 1 & \\
\hline Diminuiu & $313(50,6)$ & $149(47,6)$ & $1,28(1,06-1,55)$ & 0,026 \\
\hline Aumentou & $14(2,3)$ & $7(50)$ & $1,35(0,71-2,31)$ & \\
\hline Transtorno mental diagnosticado & & & & \\
\hline Não & $477(77,1)$ & $153(32,1)$ & 1 & \\
\hline Sim & $142(22,9)$ & $111(78,2)$ & $2,43(2,02-2,93)$ & $<0,001$ \\
\hline
\end{tabular}

IC95\%: intervalo de 95\% de confiança; RP: razão de prevalência. * Valor de p do teste de qui-quadrado de Pearson ou Teste de Fisher. Fonte: Autoria própria (2021).

Nas variáveis relacionadas à COVID-19, foi significativa a associação entre TMC e saída de casa durante o distanciamento social, rotina afetada no distanciamento social, modalidade atual de trabalho, satisfação com o modo como as 
informações sobre a COVID-19 são divulgadas pelas autoridades públicas de saúde e preocupação com a influência que a COVID-19 terá sobre os estudos. Nessas variáveis, encontrou-se maior prevalência de TMC em participantes que não estavam saindo de casa $(48 ; 53,9 \%)$, tiveram a rotina muito afetada pelo distanciamento social $(168 ; 55,8 \%)$, que não estavam trabalhando atualmente (122; 55,5\%), estavam muito insatisfeitos com o modo como as informações sobre a COVID-19 são divulgadas pelas autoridades públicas de saúde $(85 ; 57,8 \%)$ e muito preocupados com a influência que a COVID-19 terá sobre os estudos (141; 57,8\%). A Tabela 2 exibe a associação entre as variáveis sobre a COVID-19, distanciamento social e a suspeita de TMC.

Tabela 2: Associação entre variáveis sobre a COVID-19, distanciamento social e suspeita.

\begin{tabular}{|c|c|c|c|c|}
\hline Variável & $\frac{N}{n(\%)}$ & $\begin{array}{c}\text { TMC } \\
\text { n }(\%)\end{array}$ & RP $(\mathbf{I C 9 5 \%})$ & p-valor* \\
\hline \multicolumn{5}{|l|}{ Testagem para a COVID-19 } \\
\hline Não fez teste & $530(85,6)$ & $229(43,2)$ & 1 & \multirow{2}{*}{0,493} \\
\hline Fez teste & $89(14,4)$ & $35(39,3)$ & $0,91(0,68-1,18)$ & \\
\hline \multicolumn{5}{|l|}{ Resultado do teste } \\
\hline Negativo & $67(75,3)$ & $26(38,8)$ & 1 & \multirow{3}{*}{0,850} \\
\hline Positivo & $18(20,2)$ & $8(44,4)$ & $1,14(0,58-2,08)$ & \\
\hline Não sabe & $4(4,5)$ & $1(25)$ & $0,64(0,07-2,32)$ & \\
\hline \multicolumn{5}{|c|}{ Saída de casa durante o distanciamento social } \\
\hline Não sai de casa & $89(14,4)$ & $48(53,9)$ & 1 & \multirow{5}{*}{$<0,001$} \\
\hline 1 a 2 vezes na semana & $219(35,4)$ & $115(52,5)$ & $0,97(0,75-1,26)$ & \\
\hline 3 a 4 vezes na semana & $66(10,7)$ & $23(34,8)$ & $0,64(0,43-0,93)$ & \\
\hline 4 a 5 vezes na semana & $75(12,1)$ & $30(40)$ & $0,74(0,52-1,04)$ & \\
\hline Mais de 5 vezes na semana & $170(27,5)$ & $48(28,2)$ & $0,52(0,38-0,71)$ & \\
\hline \multicolumn{5}{|c|}{ Rotina afetada pelo distanciamento social } \\
\hline Não afetou & $12(1,9)$ & $2(16,7)$ & 1 & \multirow{5}{*}{$<0,001$} \\
\hline Afetou pouco & $64(10,3)$ & $16(25)$ & $1,50(0,55-5,61)$ & \\
\hline Não sei responder & $15(2,4)$ & $6(40)$ & $2,40(0,77-9,59)$ & \\
\hline Afetou & $227(36,7)$ & $72(31,7)$ & $1,90(0,76-6,88)$ & \\
\hline Afetou muito & $301(48,6)$ & $168(55,8)$ & $3,34(1,35-12,05)$ & \\
\hline \multicolumn{5}{|l|}{ Modalidade atual de trabalho } \\
\hline Trabalho presencial & $223(36)$ & $69(30,9)$ & 1 & \multirow{3}{*}{$<0,001$} \\
\hline Não está trabalhando atualmente & $220(35,5)$ & $122(55,5)$ & $1,79(1,43-2,24)$ & \\
\hline Teletrabalho & $176(28,4)$ & $73(41,5)$ & $1,34(1,04-1,72)$ & \\
\hline \multicolumn{5}{|c|}{$\begin{array}{l}\text { Satisfação com o modo como as informações sobre a COVID-19 são divulgadas pelas autoridades } \\
\text { públicas de saúde }\end{array}$} \\
\hline Muito insatisfeito & $147(23,7)$ & $85(57,8)$ & 1 & \multirow{5}{*}{$<0,001$} \\
\hline Insatisfeito & $191(30,9)$ & $84(44)$ & $0,76(0,60-0,95)$ & \\
\hline Indiferente & $93(15)$ & $32(34,4)$ & $0,59(0,43-0,80)$ & \\
\hline Satisfeito & $162(26,2)$ & $52(32,1)$ & $0,55(0,42-0,72)$ & \\
\hline Muito satisfeito & $26(4,2)$ & $11(42,3)$ & $0,73(0,73-1,14)$ & \\
\hline \multicolumn{5}{|c|}{ Preocupação com a influência que a COVID-19 terá sobre a economia do Brasil } \\
\hline Muito despreocupado & $15(2,4)$ & $6(40)$ & $0,99(0,49-1,74)$ & \multirow{5}{*}{0,076} \\
\hline Despreocupado & $10(1,6)$ & $6(60)$ & $1,48(0,74-2,62)$ & \\
\hline Indiferente & $42(6,8)$ & $26(61,9)$ & $1,53(1,10-2,08)$ & \\
\hline Preocupado & $193(31,2)$ & $81(42)$ & $1,03(0,84-1,27)$ & \\
\hline Muito preocupado & $359(58)$ & $145(40,4)$ & 1 & \\
\hline \multicolumn{5}{|c|}{ Preocupação com a influência que a COVID-19 terá sobre os estudos } \\
\hline Muito despreocupado & $45(7,3)$ & $19(42,2)$ & $0,73(0,49-1,03)$ & \multirow{5}{*}{$<0,001$} \\
\hline Despreocupado & $44(7,1)$ & $9(20,5)$ & $0,35(0,20-0,57)$ & \\
\hline Indiferente & $126(20,4)$ & $36(28,6)$ & $0,49(0,37-0,64)$ & \\
\hline Preocupado & $160(25,8)$ & $59(36,9)$ & $0,63(0,50-0,80)$ & \\
\hline Muito preocupado & $244(39,4)$ & $141(57,8)$ & 1 & \\
\hline
\end{tabular}

IC95\%: intervalo de 95\% de confiança; RP: razão de prevalência. * Valor de p do teste de qui-quadrado de Pearson ou Teste de Fisher. Fonte: Autoria própria (2021). 
A análise multivariada da regressão de Poisson demonstrou que a frequência de TMC nas mulheres foi maior em relação aos homens ( $\mathrm{RP}=1,68$; IC95\%: 1,32-2,16). Os participantes que moravam com mais de 5 pessoas tiveram frequência de TMC maior em relação àqueles que moravam sozinhos $(\mathrm{RP}=1,53$; IC95\%: 1,03-2,32). Participantes com um transtorno mental diagnosticado apresentaram frequência de TMC quase duas vezes maior do que os participantes que não tinham um transtorno mental (RP= 1,83; IC95\%: 1,50-2,24). Frequências menores de TMC foram encontradas na faixa etária de 46 a 59 anos em relação aos jovens de 18 a 24 anos ( $\mathrm{RP}=0,57$; IC95\%: 0,39-0,81); nos participantes satisfeitos com as informações divulgadas pelas autoridades públicas de saúde em relação aos muito insatisfeitos ( $\mathrm{RP}=0,75$; IC95\%: 0,56-0,99) e naqueles que estavam despreocupados $(\mathrm{RP}=0,49$; IC95\%: 0,27-0,81) ou indiferentes $(\mathrm{RP}=0,63$; IC95\%: 0,46-0,84) com a influência da COVID-19 sobre os estudos em relação àqueles que estavam muito preocupados. A Tabela 3 apresenta o modelo final hierarquizado dos fatores associados à TMC.

Tabela 3: Modelo final hierarquizado dos fatores associados à TMC.

\begin{tabular}{|c|c|c|c|c|c|c|c|c|}
\hline \multirow[b]{2}{*}{ Variável } & \multicolumn{4}{|c|}{ Modelo 1} & \multicolumn{4}{|c|}{ Modelo 1 + Modelo 2} \\
\hline & \multirow[t]{2}{*}{$\mathbf{R P}$} & \multicolumn{2}{|c|}{ IC95\% } & \multirow[t]{2}{*}{ p-valor } & \multirow[t]{2}{*}{$\mathbf{R P}$} & \multicolumn{2}{|c|}{ IC95\% } & \multirow[t]{2}{*}{ p-valor* } \\
\hline \multicolumn{5}{|l|}{ Faixa etária } & & & & \\
\hline $18-24$ & 1 & & & & 1 & & & \\
\hline $25-32$ & 0,91 & 0,69 & 1,19 & 0,496 & 0,95 & 0,74 & 1,22 & 0,728 \\
\hline $33-45$ & 0,71 & 0,48 & 1,02 & 0,092 & 0,77 & 0,57 & 1,03 & 0,088 \\
\hline $46-59$ & 0,47 & 0,29 & 0,757 & 0,002 & 0,57 & 0,39 & 0,81 & 0,003 \\
\hline $60-72$ & 0,51 & 0,22 & 1,09 & 0,103 & 0,68 & 0,31 & 1,30 & 0,291 \\
\hline \multicolumn{9}{|l|}{ Sexo } \\
\hline Feminino & 1,67 & 1,31 & 2,17 & $<0,001$ & 1,68 & 1,32 & 2,16 & $<0,001$ \\
\hline Masculino & 1 & & & & 1 & & & \\
\hline \multicolumn{9}{|l|}{ Moradia } \\
\hline Moro sozinho & 1 & & & & 1 & & & \\
\hline 02 pessoas & 1,13 & 0,76 & 1,71 & 0,542 & 1,11 & 0,76 & 1,66 & 0,600 \\
\hline 03 pessoas & 1,07 & 0,72 & 1,62 & 0,741 & 1,08 & 0,74 & 1,61 & 0,693 \\
\hline 04 pessoas & 1,18 & 0,80 & 1,80 & 0,403 & 1,15 & 0,79 & 1,72 & 0,462 \\
\hline 5 ou mais pessoas & 1,67 & 1,09 & 2,58 & 0,019 & 1,53 & 1,03 & 2,32 & 0,039 \\
\hline \multicolumn{9}{|c|}{ Transtorno mental diagnosticado } \\
\hline Não & 1 & & & & 1 & & & \\
\hline Sim & 1,93 & 1,58 & 2,35 & $<0,001$ & 1,83 & 1,50 & 2,24 & $<0,001$ \\
\hline \multicolumn{9}{|c|}{$\begin{array}{l}\text { Satisfação com o modo como as informações sobre a COVID-19 são divulgadas pelas autoridades } \\
\text { públicas de saúde }\end{array}$} \\
\hline Muito insatisfeito & & & & & 1 & & & \\
\hline Insatisfeito & & & & & 0,86 & 0,68 & 1,10 & 0,242 \\
\hline Indiferente & & & & & 0,83 & 0,59 & 1,14 & 0,267 \\
\hline Satisfeito & & & & & 0,75 & 0,56 & 0,99 & 0,050 \\
\hline Muito satisfeito & & & & & 0,79 & 0,47 & 1,26 & 0,358 \\
\hline \multicolumn{9}{|c|}{ Preocupação com a influência da COVID-19 sobre os estudos } \\
\hline Muito despreocupado & & & & & 1,08 & 0,72 & 1,56 & 0,691 \\
\hline Despreocupado & & & & & 0,49 & 0,27 & 0,81 & 0,010 \\
\hline Indiferente & & & & & 0,63 & 0,46 & 0,84 & 0,003 \\
\hline Preocupado & & & & & 0,78 & 0,61 & 1,00 & 0,054 \\
\hline Muito preocupado & & & & & 1 & & & \\
\hline
\end{tabular}

IC95\%: intervalo de 95\% de confiança; RP: razão de prevalência. * Valor de p da regressão múltipla de Poisson com variâncias robustas. Fonte: Autoria própria (2021).

\section{Discussão}

Os dados desta pesquisa demonstraram a prevalência de TMC em uma amostra da população mineira durante um período da pandemia da COVID-19 e do distanciamento social no momento em que o estado de Minas Gerais contabilizava 
174.402 casos confirmados, 4.132 óbitos e percentual médio de distanciamento social variando de 35,01\% (julho/2020) a 42,22\% (agosto/2020) (Secretaria de Estado de Saúde de Minas Gerais, 2021). Não havia vacina disponível no período pesquisado.

A ocorrência da pandemia gerou um impacto psicológico nesta amostra de habitantes do estado de Minas Gerais, principalmente nos participantes jovens, solteiros, mulheres, que tiveram a rotina, os estudos e a renda familiar afetada e estavam muito insatisfeitos com o modo como as informações sobre a COVID-19 foram divulgadas pelas autoridades públicas de saúde e muito preocupados com a influência que a COVID-19 terá sobre os estudos. Deve-se considerar, todavia, um possível viés na amostragem, posto a predominância de participantes jovens e de estudantes universitários. Apesar disso, foi possível comparar os dados encontrados com a literatura nacional e internacional sobre o tema.

Uma revisão sistemática com pesquisas na China, Espanha, Itália, Irã, Estados Unidos, Turquia, Nepal e Dinamarca, demonstrou que indivíduos considerados jovens (abaixo de 40 anos) estiveram associados à depressão e ansiedade durante a ocorrência da pandemia da COVID-19 (Xiong et al., 2020). No Brasil, também durante a pandemia, os jovens também estão mais suscetíveis à depressão, ansiedade e estresse (Goularte et al., 2021) além de exibirem risco 6\% maior de apresentar transtornos mentais menores (Duarte et al., 2020).

Em relação à maior prevalência de TMC no sexo feminino, uma pesquisa com amostra do estado do Rio Grande do Sul demonstrou que as mulheres possuem quase três vezes mais chances de risco para transtornos mentais menores no contexto da pandemia (Duarte et al., 2020). Alguns dos elementos que podem contribuir para esta maior chance estão relacionados com a violência doméstica, a divisão desigual das tarefas de casa e do cuidado dos filhos (Vieira, Garcia, \& Maciel, 2020). Ressaltase, entretanto, que mesmo anterior à pandemia, os transtornos mentais tendem a ser mais prevalentes nas mulheres, possivelmente devido a fatores hormonais, psicológicos, características dos instrumentos de avaliação e métodos de pesquisa (É. G. dos Santos \& Siqueira, 2010).

Parte significativa da amostra desta pesquisa foi composta por estudantes universitários. Esses estudantes apresentaram significativo sofrimento mental no contexto da pandemia, estando esse sofrimento associado à grande preocupação em relação aos estudos. Desde o início da pandemia as aulas presenciais de diversas universidades brasileiras foram temporariamente suspensas e substituídas por aulas em meios digitais (Brasil. Ministério da Educação, 2020). A preocupação que afeta a saúde mental dos estudantes pode estar relacionada à dificuldade de acesso à internet para acompanhar as aulas online, às mudanças no processo de ensino-aprendizagem e às dificuldades de realizar estágios práticos mantendo-se as normas do distanciamento social. Comparando com o período anterior à pandemia, há evidências de um aumento significativo de ansiedade, depressão e estresse entre os estudantes universitários durante o período pandêmico (Maia \& Dias, 2020). Esse aumento é preocupante porque anteriormente à pandemia já se identificava prevalências de TMC maiores em estudantes universitários em relação à população geral, sendo os fatores de risco relacionados à vida acadêmica e à saúde (Graner \& Cerqueira, 2019).

Em relação à ocupação profissional e à modalidade de trabalho, os participantes desta pesquisa que não estavam trabalhando demonstraram maior impacto psicológico. No período de coleta desta pesquisa, a taxa de desocupação em Minas Gerais foi de 12,2\% em julho e 12,3\% em agosto, um aumento em relação a maio de 2020 (10,4\%) quando se iniciou a Pesquisa Nacional por Amostra de Domicílios COVID-19 (Pnad COVID-19), mas ainda assim abaixo da taxa nacional de 13,1\% em julho e 13,6\% em agosto (Brasil. Instituto Brasileiro de Geografia e Estatística, 2020). A ausência de renda neste momento da pandemia pode ser um grande estressor, uma vez que dificulta o cumprimento de orientações sanitárias e o acesso a serviços de saúde (Costa, 2020). Na população geral, estar ou perceber o risco de estar desempregado durante a pandemia foi considerado um fator de risco para depressão (Xiong et al., 2020). Considera-se importante, portanto, a elaboração de políticas públicas para a manutenção do emprego e da remuneração dos trabalhadores.

Além do impacto psicológico do desemprego neste momento da pandemia, os profissionais de saúde que estão na linha de frente encontram-se entre os grupos que despertam preocupação em relação à saúde mental. Os profissionais de saúde mineiros 
que estão na linha de frente demonstraram menor prevalência de TMC nesta pesquisa. Acredita-se que o tamanho da amostra e o período de coleta dos dados podem ser fatores que influenciaram este resultado, posto que outras pesquisas demonstraram que os profissionais de saúde apresentam significativas prevalências de depressão, ansiedade, estresse e problemas para dormir (Brito-Marques, Franco, Brito-Marques, Martinez, \& Prado, 2021; Danet Danet, 2021). Uma pesquisa com médicos brasileiros constatou 49,79\% de prevalência de TMC, principalmente nos profissionais que trabalhavam no serviço público (Pimentel et al., 2020).

A diminuição na renda declarada pelos participantes mineiros foi proporcional à diminuição declarada pelos participantes da pesquisa de Almeida et al. (2020), que, comparando com o período anterior à pandemia, constataram que 55,1\% dos participantes tiveram diminuição na renda e 7\% ficaram totalmente sem rendimentos. O distanciamento social possui impactos diferentes dependendo da renda do indivíduo. Pessoas com maior renda citam impactos no convívio social, enquanto que pessoas de baixa renda citam impactos financeiros (Bezerra, da Silva, Soares, \& da Silva, 2020). Confirmando o impacto psicológico nos participantes desta pesquisa que tiveram a renda afetada, possuir baixar renda também esteve associado ao surgimento de sintomas psiquiátricos como ansiedade, depressão e transtorno de estresse pós-traumático (Goularte et al., 2021).

Em relação à saúde mental, enquanto os mineiros majoritariamente sentiram-se nervosos, tensos ou preocupados, uma pesquisa nacional constatou que $40,4 \%$ dos brasileiros sentiram-se tristes ou depressivos e 52,6\% sentiram-se ansiosos e nervosos (Barros et al., 2020). Adicionalmente, ter um transtorno mental constituiu-se fator de risco para ter a saúde mental prejudicada durante a pandemia (Barros et al., 2020; Duarte, Santo, Lima, Giordani, \& Trentini, 2020), o que corrobora a associação encontrada nesta pesquisa entre ter um transtorno mental e suspeita de TMC no período da pandemia pesquisado. Embora esse resultado seja esperado devido à própria sensibilidade do instrumento de pesquisa, os estressores originados na pandemia podem contribuir para agravar o quadro de saúde mental dos indivíduos (Duarte et al., 2020). Outros sintomas do SRQ-20 citados pelos participantes mineiros como dormir mal, achar difícil tomar decisões e ficar cansado com facilidade assemelham-se à sintomatologia de outras pesquisas da literatura nacional (Barros et al., 2020; C. B. Bezerra et al., 2020; Malta et al., 2020), mas a proporção de pessoas sentindo-se nervosas foi maior entre os mineiros do que o relatado na pesquisa nacional de C. B. Bezerra et al. (2020).

O distanciamento social é uma medida não farmacológica empregada com o objetivo de reduzir o contato físico entre as pessoas (Aquino, Silveira, Pescarini, Aquino, \& de Souza-Filho, 2020). Conforme demonstrado nesta pesquisa, não sair de casa pode trazer algum impacto psicológico ao indivíduo. Esse impacto pode ser explicado devido à limitação da mobilidade, à falta de contato com familiares, à ausência de atividades coletivas de lazer, educação e trabalho e à diminuição ou perda da renda familiar devido às consequências financeiras da pandemia. Ressalta-se que o distanciamento social não é, por si só, um fator de risco para o adoecimento mental, mas sim que há influência de outros fatores que permeiam esse contexto (Duarte et al., 2020). Deste modo, apesar dos possíveis impactos psicológicos, considera-se o distanciamento social como uma medida efetiva que diminui as chances de o indivíduo contrair a COVID-19 (Fazio et al., 2021), especialmente quando combinada ao isolamento de casos e à quarentena dos contatos (Aquino et al., 2020).

A alteração na rotina das pessoas também provocou sofrimento mental neste período da pandemia. Em uma pesquisa nacional, 76,8\% dos participantes afirmaram que o distanciamento social modificou sua rotina, mas que conseguiram se adaptar à nova realidade (C. B. Bezerra et al., 2020). Uma vez que os dados desta pesquisa foram coletados em uma fase inicial da pandemia no estado de Minas Gerais, possivelmente a população ainda se adaptava ao novo contexto da pandemia e das normas do distanciamento social.

Durante uma pandemia, desenvolver campanhas para informar e orientar a população pode ser uma importante estratégia para conter o contágio e evitar pânico na população. A insatisfação dos participantes desta pesquisa com as informações transmitidas pelas autoridades públicas de saúde pode demonstrar uma carência de informações adequadas durante o período 
pesquisado. A ausência de uma fonte confiável de informação pode abrir espaço para o surgimento de notícias falsas que contribuem para criar pânico, minar a confiança na ciência, nos trabalhadores da saúde e nas recomendações de saúde pública. Sendo assim, manter a confiança do público nas autoridades de saúde, tanto em sua resposta quanto em comunicações, é algo essencial durante um surto para que a população adote um comportamento protetor adequado à situação (Lim et al., 2020).

A pandemia da COVID-19 e o distanciamento social promoveram relevante impacto psicológico nesta amostra da população mineira. Embora não se possa afirmar que esse impacto possuiu significância clínica, considera-se importante que os profissionais e os serviços de saúde estejam preparados para receber demandas por atendimentos psicológicos.

\section{Considerações Finais}

Os dados desta pesquisa demonstram um relevante impacto psicológico nesta amostra da população do estado de Minas Gerais. Encontrou-se associações significativas entre TMC e variáveis sociodemográficas, sobre a COVID-19 e o distanciamento social. Esses resultados são importantes para compreender como a saúde mental da população é afetada durante uma pandemia.

As limitações desta pesquisa foram principalmente a amostragem por conveniência e a participação apenas de pessoas que possuíam acesso à internet e habilidades para responderem um questionário online. Deste modo, não é possível generalizar os resultados encontrados para outras populações. Enfatiza-se, entretanto, que a coleta de dados via internet foi o método mais adequado para respeitar o distanciamento social e não expor pesquisadores e participantes ao risco de contágio pela COVID-19.

Esta pesquisa possivelmente encontra-se entre as primeiras a analisar o impacto psicológico da pandemia da COVID19 e do distanciamento social nos moradores do estado de Minas Gerais. Os resultados encontrados podem auxiliar as autoridades sanitárias a planejarem políticas públicas e a mobilizarem recursos financeiros e pessoal técnico para área de saúde mental e atenção psicossocial.

Entender o impacto psicológico de um fenômeno ainda em andamento como a pandemia da COVID-19 e aplicação do distanciamento social é uma tarefa complexa. Pesquisas posteriores poderão estudar os efeitos e as consequências do novo coronavírus e da restrição de mobilidade para propor intervenções em nível individual e coletivo a fim de mitigar os prejuízos à saúde mental da população.

\section{Referências}

Almeida, W. da S. de, Szwarcwald, C. L., Malta, D. C., Barros, M. B. de A., Souza Júnior, P. R. B. de, Azevedo, L. O., \& Silva, D. R. P. da. (2020). Mudanças nas condições socioeconômicas e de saúde dos brasileiros durante a pandemia de COVID-19. Revista Brasileira de Epidemiologia, 23 , 1-14. https://doi.org/10.1590/1980-549720200105

Aquino, E. M. L., Silveira, I. H., Pescarini, J. M., Aquino, R., \& de Souza-Filho, J. A. (2020). Medidas de distanciamento social no controle da pandemia de COVID-19: potenciais impactos e desafios no Brasil. Ciencia e Saude Coletiva, 25, 2423-2446. https://doi.org/10.1590/1413-81232020256.1.10502020

Barros, M. B. de A., Lima, M. G., Malta, D. C., Szwarcwald, C. L., Azevedo, R. C. S. de, Romero, D., ... Gracie, R. (2020). Report on sadness/depression, nervousness/anxiety and sleep problems in the Brazilian adult population during the COVID-19 pandemic. Epidemiol.Serv.Saude, 29 (4), e2020427. https://doi.org/10.1590/s1679-49742020000400018

Bezerra, A. C. V., da Silva, C. E. M., Soares, F. R. G., \& da Silva, J. A. M. (2020). Fatores associados ao comportamento da população durante o isolamento social na pandemia de COVID-19. Ciencia e Saude Coletiva, 25, 2411-2421. https://doi.org/10.1590/1413-81232020256.1.10792020

Bezerra, C. B., Saintrain, M. V. de L., Braga, D. R. A., Santos, F. da S., Lima, A. O. P., de Brito, E. H. S., \& Pontes, C. de B. (2020). Impacto psicossocial do isolamento durante pandemia de COVID-19 na população brasileira: análise transversal preliminar. Saude $e$ Sociedade, 29(4), 1-10. https://doi.org/10.1590/S0104-12902020200412

Brasil. Instituto Brasileiro de Geografia e Estatística. (2020). Pesquisa Nacional por Amostra de Domicílios : PNAD COVID19. Retrieved July 5, 2021, from https://www.ibge.gov.br/estatisticas/sociais/trabalho/27947-divulgacao-mensal-pnadcovid2.html?edicao=28926\&t=0-que-e

Brasil. Ministério da Educação. (2020). Portaria No 343, de 17 de março de 2020. Dispõe sobre a substituição das aulas presenciais por aulas em meios digitais enquanto durar a situação de pandemia do Novo Coronavírus - COVID-19. Recuperado 10 de maio de 2021, de https://www.in.gov.br/en/web/dou/-/portaria-n343-de-17-de-marco-de-2020-248564376

Brito-Marques, J. M. de A. M., Franco, C. M. R., Brito-Marques, P. R. de, Martinez, S. C. G., \& Prado, G. F. do. (2021). Impact of COVID-19 pandemic on the sleep quality of medical professionals in Brazil. Arquivos de neuro-psiquiatria, 79(2), 149-155. https://doi.org/10.1590/0004-282X-anp-2020-0449 
Chagas, C. A., Castro, T. G. de, Leite, M. S., Viana, M. A. C. B. M., Beinner, M. A., \& Pimenta, A. M. (2019). Prevalência estimada e fatores associados à hipertensão arterial em indígenas adultos Krenak do Estado de Minas Gerais, Brasil. Cadernos de Saude Publica, 36(1), e00206818. https://doi.org/10.1590/0102-311X00206818

Costa, S. da S. (2020). The pandemic and the labor market in Brazil Simone. Revista de Administração Pública, 54(4), 969-978. https://doi.org/10.1590/0034761220200170

Coutinho, L. M. S., Scazufca, M., \& Menezes, P. R. (2008). Métodos para estimar razão de prevalência em estudos de corte transversal. Revista de Saúde Pública, 42(6), 992-998. https://doi.org/10.1590/s0034-89102008000600003

Danet Danet, A. (2021). Impacto psicológico de la COVID-19 en profesionales sanitarios de primera línea en el ámbito occidental. Una revisión sistemática. Medicina Clinica. https://doi.org/10.1016/j.medcli.2020.11.009

Duarte, M. de Q., Santo, M. A. da S., Lima, C. P., Giordani, J. P., \& Trentini, C. M. (2020). COVID-19 e os impactos na saúde mental: uma amostra do Rio Grande do Sul, Brasil. Ciencia e Saude Coletiva, 25(9), 3401-3411. https://doi.org/10.1590/1413-81232020259.16472020

Fazio, R. H., Ruisch, B. C., Moore, C. A., Granados Samayoa, J. A., Boggs, S. T., \& Ladanyi, J. T. (2021). Social distancing decreases an individual's likelihood of contracting COVID-19. Proceedings of the National Academy of Sciences of the United States of America, 118(8). https://doi.org/10.1073/pnas.2023131118

Goularte, J. F., Serafim, S. D., Colombo, R., Hogg, B., Caldieraro, M. A., \& Rosa, A. R. (2021). COVID-19 and mental health in Brazil: Psychiatric symptoms in the general population. Journal of Psychiatric Research, 132, 32-37. https://doi.org/10.1016/j.jpsychires.2020.09.021

Graner, K. M., \& Cerqueira, A. T. D. A. R. (2019). Revisão integrativa: sofrimento psíquico em estudantes universitários e fatores associados Integrative. Ciencia e Saude Coletiva, 24(4), 1327-1346. https://doi.org/10.1590/1413-81232018244.09692017

Huremovic, D. (2019). Psychiatry of Pandemics: A Mental Health Response to Infection Outbreak. Springer.

Ko, C. H., Yen, C. F., Yen, J. Y., \& Yang, M. J. (2006). Psychosocial impact among the public of the severe acute respiratory syndrome epidemic in Taiwan. Psychiatry and Clinical Neurosciences, 60(4), 397-403. https://doi.org/10.1111/j.1440-1819.2006.01522.x

Lim, V. W., Lim, R. L., Tan, Y. R., Soh, A. S. E., Tan, M. X., Othman, N. B., \& Chen, M. I. C. (2020). Government trust, perceptions of COVID-19 and behaviour change: Cohort surveys, singapore. Bulletin of the World Health Organization, 99(2), 92-101. https://doi.org/10.2471/BLT.20.269142

Maia, B. R., \& Dias, P. C. (2020). Anxiety, depression and stress in university students: The impact of COVID-19. Estudos de Psicologia (Campinas), 37. https://doi.org/10.1590/1982-0275202037e200067

Malta, D. C., Gomes, C. S., Szwarcwald, C. L., Barros, M. B. de A., Silva, A. G., Prates, E. J. S., \& Rodrigues Pereira da Silva, D. (2020). Distanciamento social, sentimento de tristeza e estilos de vida da população brasileira durante a pandemia de COVID-19. Saúde em Debate, 1-22. https://doi.org/10.1590/SciELOPreprints.1371

Mari, J. de J., \& Williams, P. (1985). A Comparison of the Validity of Two Psychiatric Screening Questionnaires (Ghq-12 and Srq-20) In Brazil, Using Relative Operating Characteristic (Roc) Analysis. Psychological Medicine, 15, 651-659. https://doi.org/10.1017/S0033291700031500

Minas Gerais. (2020). Decreto № 47.891, de 20 de março de 2020. Reconhece o estado de calamidade pública decorrente da pandemia causada pelo agente Coronavírus (COVID-19). https://www.saude.mg.gov.br/images/noticias_e_eventos/000_2020/coronavirus-legislacoes/25-03/Decreto-n-47.891-20.03.20.pdf

Santos, K. O. B., Araújo, T. M., Pinho, P. S., \& Silva, A. C. C. (2010). Avaliação de um Instrumento de Mensuração de Morbidade Psíquica. Revista Baiana de Saúde Pública, 34(3), 544-560.

Pimentel, D., Figueiredo, D. L., Mattos, R. M. P. R. de, \& Barreto, I. D. de C. (2020). Mental health of Brazilian physicians during the COVID-19 pandemic. Research, Society and Development, 9(10), e5129108758. https://doi.org/10.33448/rsd-v9i10.8758

Santos, É. G. dos, \& Siqueira, M. M. de. (2010). Prevalência dos transtornos mentais na população adulta brasileira: Uma revisão sistemática de 1997 a 2009. Jornal Brasileiro de Psiquiatria, Vol. 59, p. 238-246. https://doi.org/10.1590/S0047-20852010000300011

Secretaria de Estado de Saúde de Minas Gerais. (2021). Coronavírus SES-MG. Recuperado 12 de maio de 2021, de https://coronavirus.saude.mg.gov.br/

Sohrabi, C., Alsafi, Z., O’Neill, N., Khan, M., Kerwan, A., Al-Jabir, A., \& Agha, R. (2020, abril 1). World Health Organization declares global emergency: A review of the 2019 novel coronavirus (COVID-19). International Journal of Surgery, Vol. 76, p. 71-76. https://doi.org/10.1016/j.ijsu.2020.02.034

The Jamovi project. (2021). Jamovi (Version 1.6) https://www.jamovi.org/about.html

Vieira, P. R., Garcia, L. P., \& Maciel, E. L. N. (2020). Isolamento social e o aumento da violência doméstica: o que isso nos revela? Revista Brasileira de Epidemiologia, 23. https://doi.org/10.1590/1980-549720200033

Wang, C., Pan, R., Wan, X., Tan, Y., Xu, L., Ho, C. S., \& Ho, R. C. (2020). Immediate Psychological Responses and Associated Factors during the Initial Stage of the 2019 Coronavirus Disease (COVID-19) Epidemic among the General Population in China. International Journal of Environmental Research and Public Health, 17(5), 1729. https://doi.org/10.3390/ijerph17051729

World Health Organization. (2020). WHO Director-General's remarks at the media briefing on 2019-nCoV on 11 February 2020 . Recuperado 19 de maio de 2021, de https://www.who.int/director-general/speeches/detail/who-director-general-s-remarks-at-the-media-briefing-on-2019-ncov-on-11-february-2020

Xiong, J., Lipsitz, O., Nasri, F., Lui, L. M. W., Gill, H., Phan, L., \& Mcintyre, R. S. (2020). Impact of COVID-19 pandemic on mental health in the general population: A systematic review. Journal of Affective Disorders, 277, 55-64. https://doi.org/10.1016/j.jad.2020.08.001 